\title{
Effects of Sporisorium Reiliana Polysaccharides and Phoenix Dactylifera Monosaccharides on the Gut Microbiota and Serum Metabolism in Mice with Fructose-Induced Hyperuricaemia
}

\author{
Zhixuan Zhang \\ Ningbo University \\ Ziyan Wang \\ Ningbo University \\ Chenyang Lu \\ Ningbo University \\ Jun Zhou \\ Ningbo University \\ Jiaojiao Han \\ Ningbo University https://orcid.org/0000-0003-2821-8522 \\ Xiurong Su ( $\nabla$ suxiurong_public@163.com ) \\ Ningbo University https://orcid.org/0000-0003-2336-191X
}

\section{Research Article}

Keywords: Sphacelotheca reiliana polysaccharide, Phoenix dactylifera monosaccharide, fructose, hyperuricaemic, metagenomics, metabolomics

Posted Date: December 9th, 2021

DOI: https://doi.org/10.21203/rs.3.rs-1139547/v1

License: (c) (i) This work is licensed under a Creative Commons Attribution 4.0 International License. Read Full License 


\section{Abstract}

In recent decades, the prevalence of hyperuricaemia has increased, and dietary fructose is an important risk factor for the development of this disease. This study investigated and compared the effects of Sphacelotheca reiliana polysaccharides and Phoenix dactylifera monosaccharides on a series of physiological and biochemical indicators and on metagenomes and serum metabolites in mice with hyperuricaemia caused by a high-fructose diet. $S$. reiliana polysaccharides inhibited uric acid biosynthesis and promoted uric acid excretion, thereby alleviating the hyperuricaemia phenotype. In addition, hyperuricaemia was closely related to the gut microbiota. After treatment with $S$. reiliana polysaccharides, the abundance of Bacteroidetes and Proteobacteria in the mouse intestines was decreased, the expression of genes involved in glycolysis/gluconeogenesis metabolic pathways and purine metabolism was downregulated, and the dysfunction of the gut microbiota was alleviated. With regard to serum metabolism, the abundance of hippuric acid, uridine, kynurenic acid, propionic acid and arachidonoyl decreased, and the abundance of serum metabolites in inflammatory pathways involved in kidney injury and gout, such as bile acid metabolism, purine metabolism and tryptophan metabolism pathways, decreased. P. dactylifera monosaccharides aggravated hyperuricaemia. This research provides a valuable reference for the development of sugar applications.

\section{Introduction}

Hyperuricaemia (HUA) is a metabolic state characterized by elevated serum urate (SU) that has been confirmed to be caused by abnormal purine metabolism, and urate is the main product of purine metabolism(Lu et al. 2020). Sugars are usually divided into three categories, namely, monosaccharides, disaccharides, and polysaccharides. Fructose is a simple sugar found in fruits, vegetables and honey. Studies have shown that long-term consumption of fructose can inhibit renal uric acid excretion, leading to increased serum uric acid levels(Zgaga et al. 2012). In recent years, due to the widespread consumption of high-fructose diets, the global prevalence of hyperuricaemia has increased significantly. The current drug therapy has strong side effects, so it is necessary to find natural active substances to relieve hyperuricaemia given the trend of high-fructose diet consumption. Because disaccharides are easily decomposed in organisms, $S$. reiliana polysaccharides and $P$. dactylifera monosaccharides were selected as important research objects in this study.

S. reiliana is a fungus with good nutritional value and medicinal value that is responsible for head smut disease in sorghum(Prom et al. 2021). Through study of its components, it has been found that this fungus contains rich active ingredients that can be used as functional foods to produce biologically active substances such as protein, vitamins, polysaccharides and minerals. It is a kind of health food with good value for nutrition and medicine. Fungal polysaccharides are natural macromolecular compounds derived from the fruit bodies or mycelia of fungi. With the continuous deepening of polysaccharide research, it has gradually been discovered that polysaccharides from different plant sources have antiviral properties, anticancer immune-regulating effects, blood sugar- and blood fatlowering effects, anti-ageing effects and many other functions(Kimura et al. 2006; Yue et al. 2018). 
Therefore, they have been developed as a variety of drugs and food additives (such as lentinan, Hericium polysaccharides, Ganoderma lucidum polysaccharides, brown seaweed polysaccharides, white fungus polysaccharides and black fungus polysaccharides). At present, there is much research on planting, preservation and storage, but there have been few domestic studies on the functions of $S$. reiliana. $P$. dactylifera has long been one of the most important fruit crops in the arid regions of the Arabian Peninsula, North Africa, and the Middle East(A et al. 2005). P. dactylifera varieties are rich in sugar, fibre and minerals (potassium, magnesium and calcium), and the content of fructose is as high as $90 \%$, but $P$. dactylifera has a low content of protein, amino acids (methionine, tyrosine and phenylalanine) and lipids (Assirey and Rahman 2015). In addition, it is rich in phenols, flavonoids, carotenoids, etc., and has antioxidant, antimutagenesis and medicinal value (Tang et al. 2013). At present, there are few studies on the relationship between $P$. dactylifera and fructose-induced hyperuricaemia. High-fructose-related hyperuricemia has been analysed from the perspectives of physiology and biochemistry, but it has not been analysed from the perspectives of the microbiota and serum metabolism.

Diet has been shown to have dramatic effects on the gut microbiota and thereby to affect host metabolism. Studies have found that increased abundance of Bacteroides caccae and decreased abundance of Bifidobacterium pseudocatenulatum occur during the onset of hyperuricaemia(Shao et al. 2017); that corresponding butyric acid synthesis, vitamin and coenzyme metabolism functions are reduced; that branched-chain amino acid transport functions are enhanced; and that the composition of the gut microbiota of healthy people is different. Due to the existence of various associations between various diseases and the gut microbiota, treatments targeting them have been continuously developed in recent years(Zakaria 2020).

Among serum metabolites are many biomarkers and substances that cause various diseases. Some specific compounds are endogenous, including metabolites that are strongly inherited or influenced by gut microbes and others that are influenced by lifestyle factors(Marchand et al. 2016). Fructose-induced hyperuricaemia is also related to metabolite changes. After consumption of a large amount of fructose, a series of enzymes induce change during fructose metabolism and transformation, leading to abnormal uric acid metabolism and changes in various metabolic indicators; metabolic syndrome even occurs in severe cases. Compared with individuals without hyperuricaemia, patients with hyperuricaemia have different changes in the intestinal microflora and serum metabolites.

It is of great practical significance for early clinical prevention and treatment to select animal models that exhibit uric acid metabolism similar to the abnormal uric acid metabolism caused by an excessively highfructose diet in humans and to carry out antagonist studies on bioactive substances against hyperuricaemia(Valarezo et al. 2019). In the present study, a fructose-induced hyperuricaemia mouse model was treated with $S$. reiliana polysaccharide and $P$. dactylifera monosaccharide diets, and a series of analyses were conducted on the physiological and biochemical variables, transcription levels, metagenomes and serum metabolite data of the mice.

\section{Materials And Methods}




\subsection{Extraction of monosaccharides and polysaccharides}

S. reiliana was purchased from the professional farmer cooperative of Lishu County (Lishu, Jilin, China). $P$. dactylifera was purchased from Dubai, United Arab Emirates. S. reiliana and P. dactylifera were extracted with hot water; $75 \%$ ethanol was used to precipitate the $S$. reiliana polysaccharides(Han et al. 2011), which were then hydrolysed into monosaccharides(Liu et al. 2021). The extracted S. reiliana and $P$. dactylifera were tested by Shanghai Weipu Chemical Technology Service Co., Ltd.

\subsection{Experimental design}

All experimental procedures and animal care were performed in accordance with the Guide for the Care and Use of Laboratory Animals prepared by the Ningbo Customs Technology Center (affiliated with the Zhejiang Laboratory Animal Common Service Platform), and all of the animal protocols were approved by the Ningbo Customs Technology Center under permit number SYXK (ZHE 2018-0003).

Fifty-six eight-week-old SPF C57BL/ 6 male mice $(21.91 \pm 1.19 \mathrm{~g})$ were purchased from Ziyuan Experimental Animal Science Technology Co., Ltd. (Hangzhou, Zhejiang, China) under licence SCXK (Zhejiang) 2019-0004 (number: 1911070205). The animals were housed in a controlled environment at $25^{\circ} \mathrm{C}$ under a $12 \mathrm{~h}$ light $/ 12 \mathrm{~h}$ dark cycle with free access to food. The mice were fed a standard chow diet purchased from Ningbo Customs Technology Center. After 1 week of acclimatization, the mice were randomly assigned to 7 groups: a blank control (CT) group, a high-fructose model (HF) group, an allopurinol-treated (HF+AL) group, a low-dose $S$. reiliana-treated (HF+UL) group, a high-dose $S$. reilianatreated $(\mathrm{HF}+\mathrm{UH})$ group, a low-dose $P$. dactylifera-treated $(\mathrm{HF}+\mathrm{DL})$ group and a high-dose $P$. dactyliferatreated $(\mathrm{HF}+\mathrm{DH})$ group, with eight mice per group; the mice in each group were kept in one cage. Except for the CT group, which was given normal drinking water, the other groups were all given $13 \%$ fructose solution for 8 weeks(Jimena et al. 2017). The mice in the CT group and HF group received $200 \mu \mathrm{L}$ of saline by oral gavage once a day. The mice in the HF+AL group were administered $20 \mathrm{mg} \cdot \mathrm{kg}^{-1} \cdot \mathrm{d}^{-1}$ allopurinol by gavage, while the mice in the other groups (the HF+UL, HF+UH, HF+DL and HF+DH groups) received $50 \mathrm{mg} \cdot \mathrm{kg}^{-1} \cdot \mathrm{d}^{-1}$ S. reiliana, $100 \mathrm{mg} \cdot \mathrm{kg}^{-1} \cdot \mathrm{d}^{-1}$ S. reiliana, $50 \mathrm{mg} \mathrm{kg}{ }^{-1} \cdot \mathrm{d}^{-1}$ P. dactylifera or $100 \mathrm{mg}$ $\mathrm{kg}^{-1} \cdot \mathrm{d}^{-1}$ P. dactylifera by gavage for 8 weeks (Fig. S1) (Naoyuki et al. 2011). The choice of dose, which was chosen according to previous studies-, was equivalent to a dose of $729.6 \mathrm{mg} / \mathrm{d}$ in a $60-\mathrm{kg}$ human( Jing et al. 2017; Reagan-Shaw 2008).

Body weight was measured every week. On the last day of the experiment, urine and stool samples were collected from each mouse and stored at $-80^{\circ} \mathrm{C}$. After the mice were anaesthetized with isoflurane, blood samples were obtained from the eye sockets, and serum was separated via centrifugation for $10 \mathrm{~min}$ at $4^{\circ} \mathrm{C}$ at $3500 \mathrm{rpm}$ and stored at $-80^{\circ} \mathrm{C}$ for biochemical tests. The mice were sacrificed by cervical dislocation, and their visceral organs were excised, weighed, and immediately stored at $-80^{\circ} \mathrm{C}$ until further analysis.

\subsection{Glucose tolerance test}


After they were raised for 8 weeks, the mice were fasted for $12 \mathrm{~h}$. First, the fasting blood glucose of the mice was measured by taking blood from the tail vein and recorded as the $0 \mathrm{~min}$ blood glucose. Then, glucose was injected into the abdominal cavity of each mouse at a dose of $0.5 \mathrm{~g} / \mathrm{kg}$. The blood glucose level was measured $30 \mathrm{~min}, 60 \mathrm{~min}$, and $120 \mathrm{~min}$ after glucose administration, and a glucose curve was drawn.

\subsection{Measurement of biochemical indices}

The serum, urine, faeces and liver uric acid levels; serum creatinine; urinary creatinine; blood urea nitrogen; and urine urea nitrogen contents were determined according to the instructions of the kits (Nanjing Jiancheng Bioengineering Institute, Nanjing, Jiangsu, China).

\subsection{Quantitative real-time PCR (qPCR)}

RNA extraction and qPCR analysis were performed according to previously described methods (Huang et al. 2019). Total RNA was extracted from kidney and liver tissue, and cDNA was synthesized with a TransZol Up Plus RNA Kit and a TransScript All in-One First-Strand cDNA Synthesis SuperMix Kit (TransGen Biotech, Beijing, China). A Rotor-Gene 6000 real-time PCR detection system (Rotor-Gene 6000, Qiagen, Berlin, Germany) was used for qPCR amplification. Relative quantification was performed using the $2^{-\triangle \Delta C t}$ method. The qPCR primers used in this study are presented in Table S1. $\beta$-Actin was used as an internal control(Livak and Schmittgen 2001).

\subsection{Metagenomic sequencing}

DNA samples were extracted from stool, and their purity was checked to ensure quality. Genomic DNA was sheared by ultrasound; $1 \mu \mathrm{g}$ of DNA per sample was used as sample input, and the DNA was fragmented to a size of $300 \mathrm{bp}$ for establishment of the DNA libraries. The DNA fragments were then tailed with adaptor sequences, PCR amplification was performed, and metagenomic shotgun sequencing was performed using an Illumina HiSeq 2500 (Illumina, Inc., San Diego, CA, USA)(Wang et al. 2019) at LcBio Technologies (Hangzhou) Co., Ltd. The sequence had been deposited at NCBI Sequence Read Archive Database under the accession number SRP199103.

\subsection{Serum metabolite detection}

Twenty microlitres of serum was added to $120 \mu \mathrm{L}$ of $50 \%$ methanol, shaken and mixed thoroughly, and the mixture was allowed to stand for $10 \mathrm{~min}$ at room temperature. The mixture was extracted overnight at $-20^{\circ} \mathrm{C}$ to precipitate the protein in the serum. After centrifugation, the supernatant metabolite extract was transferred to a 96-well plate. Finally, $10 \mu \mathrm{L}$ volumes from each sample were mixed into Quality Control (QC) samples. Metabolic profiling analysis of the tissues was conducted on an ultra-performance liquid chromatography (UPLC) system (SCIEX, Warrington, UK) with an ACQUITY UPLC T3 column (100 mm 2.1 $\mathrm{mm}, 1.8 \mu \mathrm{m}$, Waters, London, UK). The Q-TOF was operated separately in both the electrospray ionizationpositive (ESI+) and electrospray ionization-negative (ESI) modes. The sequencing was completed by LCBio Technologies (Hangzhou) Co., Ltd.

\subsection{Statistical analysis}


The data are shown as the means \pm standard deviations (SDs). Using SPSS 20.0 software, one-way analysis of variance (ANOVA) was used to analyse the significant differences between the groups. The data that conformed to a normal distribution were analysed by ANOVA and Tukey's post-hoc test, and the data that did not meet the assumptions of the analysis of variance were analysed by the Mann-Whitney test(Gabriel et al. 2016). GraphPad Prism 7 was used for mapping, and T tests and one-way analysis of variance were used for comparisons between groups. Differences were considered statistically significant when $p<0.05$.

\section{Results}

\subsection{Composition of $S$. reiliana polysaccharides and $P$. dactylifera monosaccharides}

Among the monosaccharide components of the extracted $S$. reiliana polysaccharides, the glucose content was the highest, reaching $55.52 \%$. The levels of mannose and galactose were $2 \%$ and $0.67 \%$, respectively (Table S2). In the extracted $P$. dactylifera monosaccharides, the glucose content was as high as $49.25 \%$, the galacturonic acid content was $0.58 \%$, the proportion of rhamnose monohydrate was $0.52 \%$, and the content of mannose was $0.45 \%$ (Table S3).

\subsection{Physiological and biochemical indices of hyperuricaemic mice}

As shown in Fig. S2a, the effects of polysaccharides and monosaccharides on glucose homeostasis in high-fructose diet-fed mice were evaluated by intraperitoneal glucose tolerance tests. After the mice were injected with glucose in the abdominal cavity, the blood glucose level of each group reached the highest at 30 min and returned to the initial level at $60 \mathrm{~min}$. The CT group had the lowest fasting blood glucose level and the largest change in blood glucose level, and the HF group had the highest blood glucose level. The blood glucose levels of the HF+AL, HF+UL and HF+UH groups had a little change, while those of the $\mathrm{HF}+\mathrm{DL}$ group and $\mathrm{HF}+\mathrm{DH}$ group did not change significantly. During the experiment, the mice in the $\mathrm{CT}$ group gained the most weight, with weight gain reaching $4.68 \mathrm{~g}$; the mice in the HF+DH monosaccharide group gained the least weight at approximately $3.98 \mathrm{~g}$ (Fig. S2b). Through statistical analysis of the organ indices of the liver and kidneys, it was found that the liver index and kidney index values were reduced in the HF+UL group. The liver index and kidney index of the HF+AL group were the highest, but there were no significant differences $(P>0.05)$ (Fig. S2c).

The levels of serum uric acid $(174.39 \pm 20.63 \mu \mathrm{mol} / \mathrm{L}, p<0.001)$, liver uric acid $(829.76 \pm 101.09 \mu \mathrm{mol} / \mathrm{L}$, $p<0.05)$, urine uric acid $(172.96 \pm 13.33 \mu \mathrm{mol} / \mathrm{L}, p<0.01)$, and faecal uric acid $(1247.69 \pm 146.80 \mu \mathrm{mol} / \mathrm{L}$, $p<0.01)$ in the HF group were significantly higher than those in the control group. Compared to the HF group, the HF+AL group exhibited significantly lower serum uric acid $(99.70 \pm 18.98 \mu \mathrm{mol} / \mathrm{L}, p<0.001)$, liver uric acid $(584.40 \pm 87.36 \mu \mathrm{mol} / \mathrm{L}, p<0.01)$, urine uric acid $(113.58 \pm 8.41 \mu \mathrm{mol} / \mathrm{L}, p<0.001)$ and faecal uric acid $(420.70 \pm 34.14 \mu \mathrm{mol}) / \mathrm{L}, p<0.001)$ levels. Serum uric acid $(125.71 \pm 14.65 \mu \mathrm{mol} / \mathrm{L}, p<0.001)$, liver uric 
acid $(604.07 \pm 8.81 \mu \mathrm{mol} / \mathrm{L}, p<0.01)$ and urine uric acid $(118.57 \pm 13.11 \mu \mathrm{mol} / \mathrm{L}, p<0.001)$ levels were decreased significantly in the HF+UL group. Serum uric acid $(125.71 \pm 14.65 \mu \mathrm{mol} / \mathrm{L}, p<0.001)$, liver uric acid $(604.07 \pm 82.81 \mu \mathrm{mol} / \mathrm{L}, p<0.01)$ and urine uric acid $(118.57 \pm 13.11 \mu \mathrm{mol} / \mathrm{L}, p<0.001)$ levels were decreased significantly in the HF+UL group. Faecal uric acid $(1735.83 \pm 266.44 \mu \mathrm{mol} / \mathrm{L}, p<0.01)$ levels were increased significantly in the $\mathrm{HF}+\mathrm{UH}$ group, but there were no significant differences in uric acid in the other groups. In the HF+DL group, serum uric acid $(129.87 \pm 20.72 \mu \mathrm{mol} / \mathrm{L}, p<0.01)$, liver uric acid $(683.91 \pm 73.11 \mu \mathrm{mol} / \mathrm{L}, p<0.05)$, and urine uric acid $(129.87 \pm 20.72 \mu \mathrm{mol} / \mathrm{L}, p<0.01)$ were decreased significantly, and faecal uric acid $(1643.63 \pm 237.70 \mu \mathrm{mol} / \mathrm{L}, p<0.01)$ was increased significantly. The faecal uric acid content $(1902.22 \pm 224.06 \mu \mathrm{mol} / \mathrm{L}, p<0.01)$ in the HF+DL group was significantly increased (Fig. 1a-b).

Compared with those in the CT group, the mice in the HF group had significantly higher serum creatinine $(56.30 \pm 6.79 \mu \mathrm{mol} / \mathrm{L}, p<0.001)$, blood urea nitrogen $(24.84 \pm 3.45 \mathrm{mmol} / \mathrm{L}, p<0.001)$ and urine urea nitrogen content $(330.90 \pm 67.83 \mathrm{mmol} / \mathrm{L}), p<0.01)$. Compared with those in the HF group, the mice in the HF+AL group had significantly lower serum creatinine $(38.68 \pm 2.71 \mu \mathrm{mol} / \mathrm{L}, p<0.001)$ and blood urea nitrogen $(16.99 \pm 1.92 \mathrm{mmol} / \mathrm{L}, p<0.001)$ levels but significantly higher urine urea nitrogen content $(566.57 \pm 140.19$ $\mathrm{mmol} / \mathrm{L}, p<0.01)$. Serum creatinine $(43.47 \pm 3.31 \mu \mathrm{mol} / \mathrm{L}, p<0.001)$, blood urea nitrogen $(18.02 \pm 3.36$ $\mathrm{mmol} / \mathrm{L}, p<0.01)$ and urine urea nitrogen were significantly increased $(489.11 \pm 28.75 \mathrm{mmol} / \mathrm{L}, p<0.001)$ in the $\mathrm{HF}+\mathrm{UL}$ group. Urinary urea nitrogen was significantly increased in the HF+UH group $(549.18 \pm 90.05$ $\mu \mathrm{mol} / \mathrm{L}, p<0.001)$. Serum creatinine $(41.91 \pm 3.10 \mu \mathrm{mol} / \mathrm{L}, p<0.001)$ and blood urea nitrogen $(20.37 \pm 3.61$ $\mathrm{mmol} / \mathrm{L}, p<0.05)$ in the HF+DL group were increased significantly. There were no significant differences in several indices in the HF+DH group (Fig. 1c-d).

\subsection{Expression of uric acid metabolism-related genes in hyperuricaemic mice}

As shown in Fig. 2, the qPCR test results showed that compared to those in the CT group, the transcription levels of ADA and XOD in the liver in the HF group were significantly upregulated $(p<0.01)$. In mice that received low doses of $S$. reiliana supplementation, the transcription levels of the XOD gene were restored to normal $(p<0.5)$. GLUT9, URAT1 and UMOD were significantly upregulated in the HF group $(p<0.001)$, whereas the expression of ABCG2 and MRP4 was significantly downregulated. Compared with those of mice in the HF group, the XOD gene transcription levels were significantly higher in the HF+DH group. GLUT9, URAT1 and UMOD were significantly upregulated in the HF+DL group and HF+DH group. ABCG2 and MRP4 were significantly upregulated in the HF+UL, HF+UH, HF+DL and HF+DH groups $(p<0.001)$.

\subsection{Expression of inflammatory factors in hyperuricaemic mice}

As shown in Fig. 3a-d, compared with those in the CT group, the transcription levels of inflammatory factors (IL-1 $\beta$, IL-6) and the anti-inflammatory factor TGF- $\beta$ in the HF group were significantly increased in both the liver and kidneys $(p<0.05)$. Compared with those in the HF group, the transcription levels of IL-1 $\beta$, 
IL- 6 and TGF- $\beta$ in the HF+UL and HF+UH groups were significantly decreased $(p<0.05)$. However, there were opposite trends in the HF+DL group and the HF+DH group. Similarly, compared with those in the CT group, the transcription levels of NLRP3 and Caspase- 1 in the HF group were significantly increased $(p<0.05)$. Compared with those in the HF group, the transcription levels of NLRP3 and Caspase- 1 in the $\mathrm{HF}+\mathrm{UL}, \mathrm{HF}+\mathrm{DL}$ and $\mathrm{HF}+\mathrm{DH}$ groups were significantly increased $(p<0.05)$ (Fig. 3e). Similarly, the transcription levels of MyD88, TRAF6 and NF-KB were significantly upregulated in the HF group compared with the CT group $(p<0.01)$, whereas both $\mathrm{HF}+\mathrm{UL}$ and $\mathrm{HF}+\mathrm{UH}$ treatment significantly downregulated the transcription of all these genes (Fig. 3f).

\subsection{Changes in the structure of the gut microbiota in hyperuricaemic mice}

Metagenomic sequencing results showed that a high-fructose diet can increase the diversity and abundance of the gut microbiota of mice, and the observed species index and Shannon index were highest in the HF+DL group (Table S4). Through weighted principal coordinate analysis (UniFrac PCoA) (Fig. S3), it was found that the structure of the gut microbiota of the mice in the HF+DH group was closest to that of the mice in the HF group, and the structure of the gut microbiota of the mice in the $\mathrm{HF}+\mathrm{AL}$ group was closest to that of the mice in the CT group. At the phylum level (Fig. 4a), compared with that in the HF group, the relative abundance of Bacteroides in the CT group was decreased from 67.17$58.66 \%$. The HF+UL group, HF+UH group, HF+DL group and HF+AL group all showed downward trends. Among them, the HF+UH group had the largest decrease; the abundance dropped from $67.17-34.81 \%$. The relative abundance of Bacteroides in the HF+DH group increased from 67.17-72.93\%. Compared with that in the HF group, the relative abundance of Firmicutes in the HF+DH group was decreased, and the relative abundance of Firmicutes in the other groups was increased. The abundance in the HF+UH group rose from $20.71-24.62 \%$. Compared with that in the HF group, the relative abundance of Proteobacteria in each group except for the HF+DL group was reduced.

As shown in Fig. 4b, at the genus level, the relative abundance of Muribaculum increased from $17.80 \%$ in the HF group to $21.08 \%$ in the HF+DH group and decreased from $17.80 \%$ in the HF group to $7.10 \%$ in the $\mathrm{HF}+\mathrm{UH}$ group. The relative abundance of Prevotella was highest in the HF+DH group $(17.19 \%$ and $17.36 \%$, respectively) and lowest in the HF+UH group (7.10\%). Compared with that in the model group, the maximum relative abundance of Clostridium in the HF+DL group was $9.74 \%$, while the relative abundance in the HF+UL group decreased from 13.91-6.98\%.

The data showed that at the species level, the relative abundance of Muribaculum intestinale was downregulated compared with that in the HF group, while it was the least downregulated in the HF+DH group, decreasing from $24.85-21.60 \%$. The Bacteroides acidifaciens (BA) abundance increased from $13.16 \%$ in the $\mathrm{HF}$ group to $18.15 \%$ in the $\mathrm{HF}+\mathrm{DH}$ group, while the relative abundance in the other groups was lower than that in the HF group. Between the CT group and the HF group, Lactobacillus murinus increased from $0.78-4.02 \%$, and the relative abundance dropped to $0.52 \%$ in the HF+UL group (Fig. 4c).

\subsection{Functional analysis of the gut microbiota}


As shown in Fig. 5a, the primary metabolic pathways of unigenes in each group of mice mainly included metabolism, human diseases, genetic information processing, environmental information processing, cellular processes and organic systems. The secondary metabolic pathways were enriched for 25 modules, including carbohydrate metabolism, amino acid metabolism, lipid metabolism, glycan biosynthesis and metabolism. The most abundant unigenes were related to carbohydrate metabolism (254085) and amino acid metabolism (163653).

As Fig. 5b shows, compared with that in the CT group, the abundance of unigenes related to purine metabolism, glycolysis/gluconeogenesis, fructose and mannose metabolism was increased in the HF group, but the abundance of unigenes related to the two-component system and quorum sensing was decreased in the HF group. Compared with those for the HF group, the results for the HF+AL group and the HF+UL group were exactly the opposite. The abundance of unigenes related to purine metabolism, glycolysis/gluconeogenesis, fructose and mannose metabolism, and nucleotide excision repair was decreased, while the abundance of unigenes related to the two-component system and quorum sensing were increased. These changes were more obvious in the HF+UH group than in the other groups; unigenes related to the two-component system, the abundance of flagellar assembly, fructose and mannose metabolism, bacterial chemotaxis, mannose metabolism and $A B C$ transporters were the most drastically altered. The abundance of unigenes related to purine metabolism, alanine, aspartate and glutamate metabolism, oxidative phosphorylation and pyruvate metabolism was lower in the HF+DL group than in the other groups. However, in the HF+DH group, unigenes related to the two-component system, ABC transporters, flagellar assembly, and cyanoamino acid metabolism were the least abundant.

The metabolic pathways associated with 82 differentially expressed genes and the corresponding species were screened, as shown in Fig. $5 \mathrm{c}$. The species corresponding to Unigene582 was Muribaculum intestinale, and the corresponding metabolic pathway was purine metabolism. The highest abundance (in the HF group) was 16154.22, and the lowest abundance (in the HF+UH group) was 5560.64.

Unigene1382 corresponded to Odoribacter splanchnicus, and the corresponding metabolic pathway was glycolysis/gluconeogenesis. The highest abundance (in the HF group) was 2392.98, while the abundance was relatively low in the HF+UL, HF+UH and HF+DL groups. The corresponding species of Unigene98 was Hungatella hathewayi, and the corresponding metabolic pathway was amino sugar and nucleotide sugar metabolism. The abundance was relatively low in the HF+UH group and was highest in the HF+DH group. The corresponding species of Unigene660 and Unigene9163 were Bacteroides vulgaris and Prevotella copri, and the corresponding metabolic pathways were phenylalanine, tyrosine and tryptophan biosynthesis and cysteine and methionine metabolism. The abundance was highest in the HF group and the HF+DH group, while the content was lowest in the HF+UH group.

\subsection{Serum metabolites in hyperuricaemic mice}

The statistics showed that 8051 and 8502 metabolites were obtained by the POS (positive) and NEG (negative) models, respectively, 4579 and 4555 of which were annotated (Table S5). PCA showed that the distribution of sample metabolites between different groups had a discrete trend, indicating that there were differences in serum metabolites after $S$. reiliana polysaccharide and $P$. dactylifera monosaccharide 
intervention (Fig. 6). The results indicated that there was a difference between the HF+DH group and the $\mathrm{HF}$ group in the pos mode or neg mode The difference between the HF+UL group and the CT group was the smallest. The difference between the HF+DH group and the HF+AL group was the largest.

As shown in Fig. 7 (Table S6-11), 30 differential metabolites were screened out in the CT group compared with the HF group, and acylcarnitine was upregulated. The abundance of sulfanilamide, trans-aconitic acid and linoleic acid was downregulated. The abundance of isonicotinic acid, propionic acid, transaconitic acid and phenyllactic acid was upregulated in the HF+AL group, while the abundance of uridine, uracil, and hippuric acid was downregulated. The secondary serum metabolites unique to the $\mathrm{HF}+\mathrm{AL}$ group were phenyllactic acid, acylcarnitine, propionic acid, uracil, L-histidine and D-ornithine. In the HF+UL group, the abundance of taurodeoxycholic acid and propionic acid was upregulated, and the abundance of taurocholic acid, uridine, and kynurenic acid was downregulated. In the HF+UH group, the abundance of trans-aconitic acid, trans-caffeic acid, propionic acid, and 2-arachidonic glycerol was upregulated, while the abundance of uridine and aniline was downregulated. The abundance of isonicotinic acid, trans-aconitic acid, and niacin in the HF+DL group was upregulated, and the serum metabolites that were downregulated included uridine, acylcarnitine, taurocholate and glycocholic acid. Unique secondary serum metabolites in the HF+DL group included glycolic acid and niacin. In the HF+DH group, the abundance of trans-aconitic acid was upregulated, while the abundance of uridine, acylcarnitine, taurocholate and phenyllactic acid was downregulated.

\subsection{KEGG pathways enriched for the different serum metabolites and the related pathways of the differential metabolites between groups}

The metabolites identified above in the positive and negative ion modes were assigned to primary metabolic pathways (Fig. 8a-b) in the KEGG database. The top priority was global and overview maps, followed by maps of lipid metabolism, amino acid metabolism, carbohydrate metabolism, and metabolism of cofactors and vitamins. The top 20 KEGG pathways of the determined serum metabolites under the positive and negative ion modes are shown in Fig. 8c-d. The serum metabolites were most enriched in metabolic pathways, followed by protein digestion and absorption, glycerophospholipid metabolism, and biosynthesis of amino acids.

Pathway analysis of patient biomarkers was performed using metaX, and the results and pathways are shown in Fig. 9. The enriched pathways of the differential metabolites in the HF group compared with the CT group (Fig. 9a) mainly included linoleic acid metabolism, arachidonic acid metabolism and biosynthesis of unsaturated fatty acids. The differential metabolites in the HF+AL group compared with the HF group (Fig. 9b) were enriched in phenylalanine metabolism, alanine, aspartate and glutamate metabolism, pyrimidine metabolism, etc. In the HF+UL group (Fig. 9c), the differential metabolites were enriched in biosynthesis of phenylpropanoids, phenylalanine metabolism and pyrimidine metabolism. The serum metabolites in the $\mathrm{HF}+\mathrm{UH}$ group were mainly enriched in phenylpropanoid biosynthesis and 
pyrimidine metabolism (Fig. 9d). In the HF+DL group (Fig. 9e), the main enriched pathways were bile acid biosynthesis, biosynthesis of phenylpropanoids and secondary bile acid biosynthesis. Serum metabolites in the HF+DH group were enriched in the secondary bile acid biosynthesis, taurine and hypotaurine metabolism and sphingolipid metabolism pathways (Fig. 9f).

\section{Discussion}

Previous studies have shown that fructose generates uric acid during its metabolism and that serum urate(Le et al. 2012) and urinary uric acid increase acutely following fructose ingestion. Continuous intake of high fructose is closely related to the occurrence and development of hyperuricaemia(Wang et al. 2016). Our study demonstrated that feeding mice a 13\% (W/V) fructose solution established a stable hyperuricaemia animal model with pathological characteristics similar to those of human hyperuricaemia. Treatment with $S$. reiliana polysaccharides significantly reduced serum uric acid levels in hyperuricaemia mice(2015). It is recognized that increased uric acid causes damage to liver and kidney functions. Metabolism of fructose increases the burden on the liver. Uric acid can be ionized into urate, and the kidneys are the main sites of urate deposition. The results also showed that the serum creatinine and urea nitrogen levels in the model group were significantly higher than those in healthy mice, and the serum creatinine and urea nitrogen levels were decreased after $S$. reiliana polysaccharide treatment. These may be some of the effects by which $S$. reiliana polysaccharides alleviate hyperuricemia, while $P$. dactylifera monosaccharides exerted the opposite effects. Multiple enzymes, including ADA and XOD, are key enzymes of purine nucleotide catabolism and play an important role in the production of uric acid in the liver(Zhu et al. 2021). The kidneys then rely on uric acid transporters to excrete uric acid. Uric acid transporters can be divided into two categories: uric acid-reabsorbing proteins (GLUT9, URAT1 and UMOD) and uric acid-secreting proteins (ABCG2 and MRP4)(Wan et al. 2019). In this study, the results suggested that $S$. reiliana polysaccharides inhibited ADA expression and promoted ABCG2 and MRP4 expression, thereby inhibiting uric acid biosynthesis and promoting excretory pathways. P. dactylifera monosaccharides promoted XOD expression and increased uric acid synthesis, which may have been the cause of the aggravation of uric acid accumulation.

An inflammatory response is a characteristic pathological feature of hyperuricaemia(Yan et al. 2015). Fructose-1-phosphoric is the first product of fructose metabolism. It is easily metabolized and transformed into glycerol triphosphate or long-chain fatty acids; triacylglycerol is then synthesized and accumulates in the liver, which promotes fatty liver, thus burdening the liver. Studies have shown that hyperuricaemia can also induce kidney disease(Ma et al. 2021). Kidney injury can lead to reduced clearance rates of creatinine and urea nitrogen, increase serum creatinine and blood urea nitrogen content, and promote the expression of pro-inflammatory cytokines and chemokines(Yang et al. 2012). Inflammatory factors (IL-1 $\beta$, IL-6, and TNF- $\alpha$ ) are closely related to the TLR4/MyD88/NF-KB signalling pathway and NLRP3 inflammasome(Tan et al. 2019). In this study, $S$. reiliana polysaccharide dietary intervention not only inhibited the activation of the NLRP3 inflammasome complex and blocked the TLR4/MyD88/NF-KB signalling pathway but also downregulated the transcription of the pro- 
inflammatory cytokines IL-1 $\beta$ and IL- 6 and upregulated the transcription of the anti-inflammatory cytokine TGF- $\beta$, thereby reducing liver and kidney inflammation.

In addition to the kidneys, the intestine is also one of the important organs involved in the excretion of uric acid, and one-third of uric acid is excreted through the intestine. Dysregulation of the gut microbiota can affect uric acid metabolism and lead to elevated serum uric acid levels. Therefore, dietary control of the gut microbiota may be a potential target for alleviating hyperuricaemia(Miao et al. 2020). Metagenomic detection of gene function is a good strategy with which to explore the interaction between the gut microbiota and the host. In terms of the species composition of the gut microbiota, we found that the gut microbiota structure of mice treated with allopurinol and $S$. reiliana polysaccharides was closer to that of healthy mice, while the structure of the gut microbiota of mice treated with $P$. dactylifera monosaccharides was closer to that of hyperuricaemia mice. Compared with healthy mice, at the phylum level, hyperuricaemia mice exhibited increased abundance of Bacteroidetes and Proteobacteria but reduced abundance of Firmicutes, which is consistent with literature reports(Yu et al. 2018). Increased Proteobacteria abundance can be regarded as a microbial marker of gut microbiota imbalance. Cardiovascular disease complications were found to be associated with Proteobacteria levels in the blood, consistent with an increased incidence of hyperuricaemia. The abundance of Proteobacteria and Bacteroidetes decreased most after treatment with $S$. reiliana polysaccharides. The increase in Bacteroidetes proportion will likely enhance the uptake of monosaccharides in the host intestinal tract and promote the synthesis of triacylglycerol in the liver, leading to insulin resistance(Ahmad et al. 2019). Yu et al.(Yu et al. 2018) found in an experiment that the abundance of several bacteria with purine absorption and uric acid decomposition functions, such as Lactobacillus and Clostridium, was decreased, which is consistent with our experimental results. However, the levels of Muribaculum, Prevotella and Muribaculum intestinale were increased among the intestinal microbes of mice treated with $S$. reiliana polysaccharides.

Muribaculum intestinale, BA and Odoribacter splanchnicus were enriched in the intestines of high fructose-treated mice. The abundance of Muribaculum intestinale was elevated in mice treated with $P$. dactylifera monosaccharides. The corresponding metabolic pathway of Muribaculum intestinale was purine metabolism, which is related to amino acid metabolism. Uric acid is an important metabolite of purine metabolism. The metabolic pathway of Odoribacter splanchnicus was glycolysis/gluconeogenesis. The abundance was highest in the hyperuricaemic mice, followed by the mice treated with $P$. dactylifera monosaccharides, and the abundance was lowest in mice treated with $S$. reiliana polysaccharides and healthy mice. In this study, uric acid levels were significantly reduced in mice treated with $S$. reiliana polysaccharides, suggesting that dietary intervention may alleviate hyperuricaemia in mice by regulating purine metabolism. BA can degrade mucin, the protective layer of the colon, and increase the production of the short-chain fatty acids acetic acid and succinic acid(Randhawa et al. 2014). Both Muribaculum intestinale and BA cause associated inflammation of the gut. Lactobacillus rhamnosus can repair intestinal inflammation and has anti-inflammatory effects; however, the abundance of Lactobacillus rhamnosus was the lowest in the hyperuricaemic mice. Gut microbiota I functional analysis showed that the abundance of purine metabolism- and glycolysis/gluconeogenesis- 
related microbes was increased in high-uric acid mice and $S$. reiliana monosaccharide-treated mice and decreased in allopurinol- and $P$. dactylifera polysaccharide-treated mice. The findings indicate that consumption of $S$. reiliana can relieve uric acid imbalance and that the gut microbiota affects the human body by causing major changes in host metabolism or substrate cometabolism.

Metabolomics mainly involves the study of the dynamic changes of all metabolites in the host body under conditions of disease or exogenous substance exposure to reflect pathophysiological changes and reveal pathogenesis. Analysis of changes in serum metabolism in the mice on a high-fructose diet revealed that similar to previous research results, the main metabolites with differences in serum were arachidonic acid, hippuric acid, uridine, uracil, kynurenic acid, etc.(Liu et al. 2011). These differential metabolites are mainly involved in glucose metabolism, the TCA cycle, tryptophan metabolism, purine metabolism, lipid metabolism, amino acid metabolism, etc.(Wang et al. 2016). After $P$. dactylifera monosaccharide treatment, the levels of bile acid metabolism, tryptophan metabolism and purine metabolism were elevated, indicating that high fructose intake is related to metabolic disorders in mice. Abnormal bile acid metabolism and levels are associated with metabolic disorders such as liver injury, diabetes, obesity, cardiovascular diseases, digestive system diseases (inflammatory bowel disease, colorectal cancer, etc.) and kidney disease (Gai et al.). It has been reported that hyperuricaemia may affect tryptophan metabolism(Perez-Pozo et al.), purine metabolism, the purine nucleotide cycle and amino acid metabolism, and uric acid is an important metabolite of purine metabolism(Liao et al. 2019). Studies have shown that kynurenic acid, an important metabolite of the tryptophan pathway, is elevated in rats with renal insufficiency. In addition, the serum of mice treated with a high-fructose diet and $P$. dactylifera monosaccharides also included markers associated with kidney injury, including uridine in the nucleotide metabolic pathway. Hippuric acid was used as a biomarker of bacterial imbalance and abnormal metabolism of the microflora(Calvani et al.). Arachidonic acid is the precursor of leukotriene biosynthesis in the body and is related to many inflammatory conditions, including gout(He et al. 2012). The expression of arachidonic acid and linoleic acid was decreased significantly in hyperuricaemic mice, while it was increased after low-dose $S$. reiliana treatment. The tricarboxylic acid cycle is an important part of energy metabolism and a pathway for the oxidation of energy substances such as sugars, fatty acids and amino acids. The levels of propionic acid, succinic acid and citric acid were decreased, suggesting abnormal energy metabolism in hyperuricaemic mice after $P$. dactylifera monosaccharide treatment.

We further explored the correlations among different flora, serum metabolites and metabolic pathways and found that a high-fructose diet resulted in metabolic disorders of succinic acid, hippuric acid and Laspartate cytidine in mice, in which carbohydrate metabolism and lipid metabolism were most involved. For example, both Bacteroides thetaiotaomicron and succinic acid were enriched in the TCA cycle. $B$. thetaiotaomicron and maleic acid were enriched in the butanoate metabolism pathway. Anaerosporobacter mobilis and uridine were enriched in the pyrimidine metabolism pathway. These results indicated that $S$. reiliana could alleviate fructose-induced hyperuricaemia from physiological and biochemical points of view. Dietary intervention changed the intestinal flora and serum metabolites of mice, thus reducing the uric acid content of the body and relieving hyperuricaemia. After eating, the 
metabolism of pyruvate and the tricarboxylic acid cycle are both in a state of decline. Pyruvate is the final product of the glycolysis pathway and is converted into lactic acid in the cytoplasm or enters the mitochondria for oxidation to produce acetyl coenzyme A, which enters the TCA cycle, thereby inhibiting the TCA cycle. This phenomenon presumably occurs because umami polysaccharides inhibit the unrestricted metabolism of fructose in the liver.

\section{Conclusion}

In summary, this study shows that $S$. reiliana polysaccharides and $P$. dactylifera monosaccharides have an effect on the occurrence of hyperuricaemia. $S$. reiliana polysaccharides can alleviate hyperuricaemia caused by fructose to a certain extent, and excessive intake of $P$. dactylifera monosaccharides can aggravate the occurrence of high uric acid. From the perspective of the populations of intestinal microbes and the levels of serum metabolites, intake of $S$. reiliana polysaccharides makes improves the flora structure and metabolite profile, while $P$. dactylifera monosaccharides exert the opposite effects. Our findings help elucidate the roles of $S$. reiliana polysaccharides and $P$. dactylifera monosaccharides in hyperuricaemia mice.

\section{Declarations}

\section{Funding}

This work was supported by the Fund of State Key Laboratory for Managing Biotic and Chemical Threats to the Quality and Safety of Agro-products (ZS20190105), and K.C. Wong Magna Fund of Ningbo University.

Conflict of interest The authors declare no competing financial interest.

\section{Author Contributions}

All authors contributed to the study conception and design. Material preparation, data collection and analysis were performed by [Zhixuan Zhang], [Ziyan Wang] and [Chenyang Lu]. The first draft of the manuscript was written by [Zhixuan Zhang], [Jiaojiao Han], [Xiurong Su] and [Jun Zhou] and all authors commented on previous versions of the manuscript. All authors read and approved the final manuscript.

\section{References}

1. Dalbeth N, Phipps-Green A, House ME, Gamble GD, Horne A, Stamp LK, Merriman TR (2015) Body mass index modulates the relationship of sugar-sweetened beverage intake with serum urate concentrations and gout. Arthritis Res Ther 17(1):263-270.

2. Abdelhak M, Guendez E, Eugene K, Panagiotis K (2005) Phenolic profile and antioxidant activity of the Algerian ripe date palm fruit ( Phoenix dactylifera ). Food Chem 89(3): 411-420. 
3. Ahmad A, Yang W, Chen G, Shafiq M, Javed S, Ali Zaidi SS, Shahid R, Liu C, Bokhari H (2019) Analysis of gut microbiota of obese individuals with type 2 diabetes and healthy individuals. PLos One 14(12):1-15.

4. Rahman A (2015) Nutritional composition of ten date palm (Phoenix dactylifera L.) cultivars fruit grown in Saudi Arabia by high performance liquid chromatography. J Taibah Univ Sci 9(1):75-79.

5. Calvani R, Miccheli A, Capuani G, Tomassini MA, Puccetti C, Delfini M, laconelli A, Nanni G, Mingrone G (2010) Gut microbiome-derived metabolites characterize a peculiar obese urinary metabotype. Int J Obes (Lond) 34(6):1095-1098.

6. Dasilva G, Pazos M, García E, Pérez J, Torres JL, Giralt M, Nogués MR, Medina I (2016) Lipidomics to analyze the influence of diets with different EPA:DHA ratios in the progression of Metabolic Syndrome using SHROB rats as a model. Food Chem 205:196-203.

7. Han Q, Yu J, Chang X, Ze J, Pu M (2011) Molecular characterization and hypoglycemic activity of a novel water-soluble polysaccharide from tea (Camellia sinensis) flower. Carbohyd Polym 86(2):797805.

8. He H, Ren X, Wang X, Shi X, Wang X, Ding Z, Gao P, Xu G (2012) Therapeutic effect of Yunnan Baiyao on rheumatoid arthritis was partially due to regulating arachidonic acid metabolism in osteoblasts. $J$ Pharm Biomed Anal 59:130-137.

9. Huang F, Zheng X, Ma X, Jiang R, Zhou W, Zhou S, Zhang Y, Lei S, Wang S, Kuang J, Han X, Wei M, You Y, Li M, Li Y, Liang D, Liu J, Chen T, Yan C, Wei R, Rajani C, Shen C, Xie G, Bian Z, Li H, Zhao A, Jia W (2019) Theabrownin from Pu-erh tea attenuates hypercholesterolemia via modulation of gut microbiota and bile acid metabolism. Nat Commun 10(1):1-17.

10. Tan J, Wan L, Chen X, Li X, Hao X, Li X, Li J, Ding H (2019) Conjugated linoleic acid ameliorates high fructose-induced hyperuricemia and renal inflammation in rats via NLRP3 inflammasome and TLR4 signaling pathway. Mol Nutr Food Res 63(12):1-9.

11. Soutelo J, Alejandra SY, Zotta E, Cecilia FM, Reyes TC, Juan PO (2017) Influence of normo-and hypogonadal condition, hyperuricemia, and high-fructose diet on renal changes in male rats. Int $J$ Endocrinol 2017:1-8.

12. Jing Y, Cai S, Ya H, Xue Z, Mei X (2017) Anti-fatigue activity of sea cucumber peptides prepared from Stichopus japonicus in an endurance swimming rat model. J Sci Food Agric 97(13):4548-4556.

13. Le MT, Frye RF, Rivard CJ, Cheng J, McFann KK, Segal MS, Johnson RJ, Johnson JA (2012) Effects of high-fructose corn syrup and sucrose on the pharmacokinetics of fructose and acute metabolic and hemodynamic responses in healthy subjects. Metabolism 61(5):641-651.

14. Liao Z, Zhang S, Liu W, Zou B, Lin L, Chen M, Liu D, Wang M, Li L, Cai Y, Liao Q, Xie Z (2019) LC-MSbased metabolomics analysis of Berberine treatment in ulcerative colitis rats. $\mathrm{J}$ Chromatogr $\mathrm{B}$ 1133:1-11.

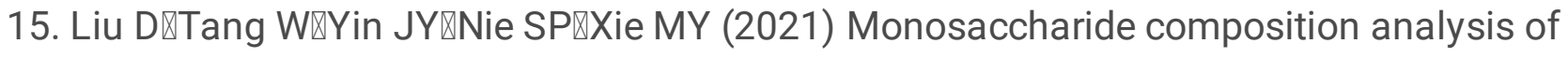
polysaccharides from natural sources: Hydrolysis condition and detection method development. Food Hydrocolloid 116(3):1-21. 
16. Liu Y, Sun X, Di D, Quan J, Zhang J, Yang X (2011) A metabolic profiling analysis of symptomatic gout in human serum and urine using high performance liquid chromatography-diode array detector technique. Clin Chim Acta 412(23-24):2132-2140.

17. Livak KJ, Schmittgen TD (2001) Analysis of relative gene expression data using real-time quantitative PCR and the $2^{-\triangle \Delta C t}$ Method. Methods 25(4):402-408.

18. Lu J, He Y, Cui L, Xing X, Liu Z, Li X, Zhang H, Li H, Sun W, Ji A, Wang Y, Yin H, Li C (2020) Hyperuricemia predisposes to the onset of diabetes via promoting pancreatic $\beta$-cell death in uricasedeficient male mice. Diabetes 69(6):1149-1163.

19. Marchand J, Martineau E, Guitton Y, Dervilly PG, Giraudeau P (2016) Multidimensional NMR approaches towards highly resolved, sensitive and high-throughput quantitative metabolomics. Curr Opin Biotechnol 43:49-55.

20. Zhang M, Zhao D, Zhou G, Li C (2020) Dietary Pattern, Gut Microbiota, and Alzheimer's Disease. J Agric Food Chem. 68(46):12800-12809.

21. Kamatani N, Fujimori S, Hada T, Hosoya T, Kohri K, Nakamura T, Ueda T, Yamamoto T, Yamanaka H, Matsuzawa Y (2011) An allopurinol-controlled, randomized, double-dummy, double-blind, parallel between-group, comparative study of febuxostat (TMX-67), a non-purine-selective inhibitor of xanthine oxidase, in patients with hyperuricemia including those with gout in Japan: phase 3 clinical study. J Clin Rheumatol 17(4 Suppl 2):13-18.

22. Perez SE, Schold J, Nakagawa T, Sánchez LG, Johnson RJ, Lillo JL (2010) Excessive fructose intake induces the features of metabolic syndrome in healthy adult men: role of uric acid in the hypertensive response. Int J Obes (Lond) 34(3):454-461.

23. Prom LK, Perumal R, Isakeit T, Erattaimuthu S, Magill C (2021) Response of Sorghum Accessions against Newly Documented Pathotypes 5 and 6 of Head Smut Pathogen Sporisorium reilianum. American Journal of Plant Sciences 12(3):432-443.

24. Liu D, Tang W, Yin JY, Nie SP, Xie MY (2021) Monosaccharide composition analysis of polysaccharides from natural sources: Hydrolysis condition and detection method development. Food Hydrocolloid 116(3):1-7.

25. Randhawa PK, Singh K, Singh N, Jaggi AS (2014) A review on chemical-induced inflammatory bowel disease models in rodents. Korean J Physiol Pharmacol 18(4):279-288.

26. Reagan-Shaw S, Nihal M, Ahmad N (2008) Dose translation from animal to human studies revisited. FASEB J 22(3):659-661.

27. Shao T, Shao L, Li H, Xie Z, He Z, Wen C (2017) Combined signature of the fecal microbiome and metabolome in patients with gout. Front Microbiol 8:268-276.

28. Tang ZX, Shi LE, Aleid SM (2013) Date fruit: chemical composition, nutritional and medicinal values, products. J Sci Food Agric 93(10):2351-2361.

29. Eduardo V, María CG, Mario P, Miguel AM (2019) Chemical composition and biological activity of the essential oil from gnaphalium elegans kunth from Loja, Ecuador. J Essent Oil Bear PI 22(5):13721378. 
30. Wan H, Han J , Tang S, Bao W , Lu C, Zhou J , Ming T, Li Y , Su X (2019) Comparisons of protective effects between two sea cucumber hydrolysates against diet induced hyperuricemia and renal inflammation in mice. Food Funct 11(1):1074-1086.

31. Wang JJ, Zhang RQ, Zhai QY, Liu JC, Li N, Liu WX, Li L, Shen W (2019) Metagenomic analysis of gut microbiota alteration in a mouse model exposed to mycotoxin deoxynivalenol. Toxicol Appl Pharmacol 372:47-56.

32. Wang Y, Zhao M, Xin Y, Liu J, Wang M, Zhao C (2016) (1)H-NMR and MS based metabolomics study of the therapeutic effect of Cortex Fraxini on hyperuricemic rats. J Ethnopharmacol 185:272-281.

33. Ding Y, Yang H, Xiang W, He X, Liao W, Yi Z (2015) CD200R1 agonist attenuates LPS-induced inflammatory response in human renal proximal tubular epithelial cells by regulating TLR4-MyD88TAK1-mediated NF-KB and MAPK pathway. Biochem Biophys Res Commun 460(2):287-294.

34. Zhou Y, Fang L, Jiang L, Wen P, Cao H, He W, Dai C, Yang J (2012) Uric Acid induces renal inflammation via activating tubular NF-KB signaling pathway. PLos One 7(6):1-10.

35. Yu Y, Liu Q, Li H, Wen C, He Z (2018) Alterations of the gut microbiome associated with the treatment of hyperuricaemia in male rats. Front Microbiol 9:1-10.

36. Gomaa EZ (2020) Human gut microbiota/microbiome in health and diseases: a review. Antonie van Leeuwenhoek 113(12):2019-2040.

37. Gai Z, Gui T, Hiller C, Kullak-Ublick GA (2016) Farnesoid X receptor protects against kidney injury in uninephrectomized obese mice. J Biol Chem 291(5):2397-2411.

38. Zhu Y, Zhang RX, Cai Y, Ma MY, Gu YG, Zhang RZ, Pan HX, Chang X (2021) Rice peptide and collagen peptide prevented potassium Oxonate-induced hyperuricemia and renal damage. Food Biosci 42(2):1-8.

\section{Figures}
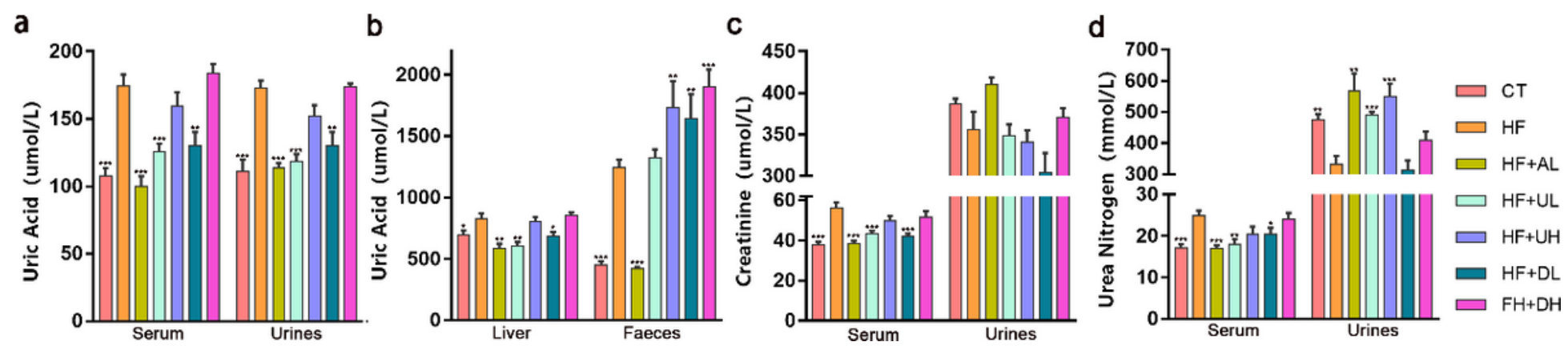

Figure 1

Effects on hyperuricaemia-related indicators. (a) Serum uric acid, urine uric acid. (b) Liver uric acid, faecal uric acid. (c) Serum creatinine, urine creatinine. (d) Blood urea nitrogen, urine urea nitrogen. The data are expressed as the mean $\pm S D, n=8$. Significant differences $(P<0.05)$ were determined by ANOVA. 

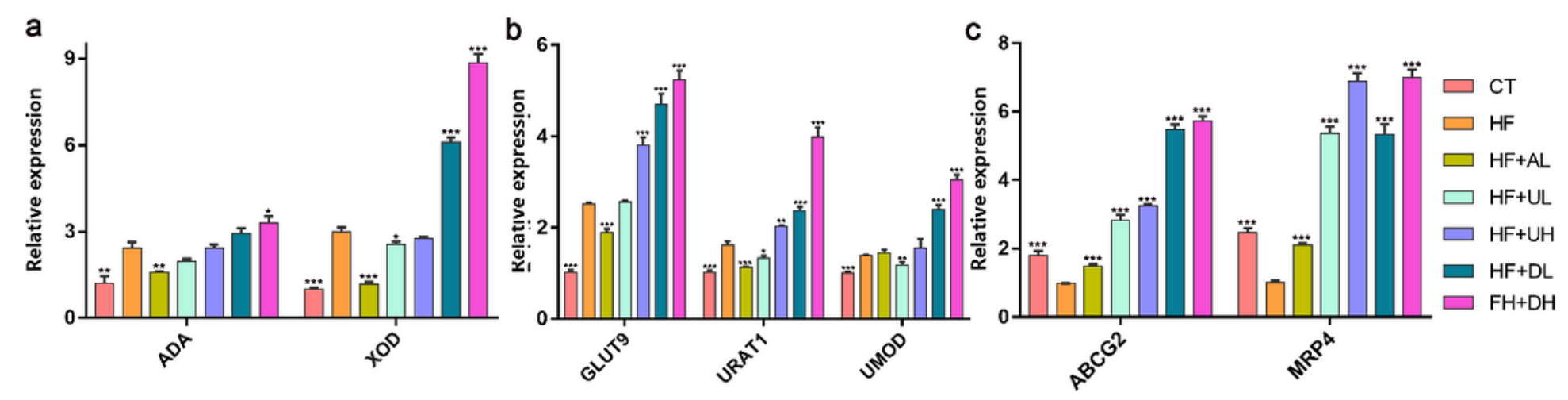

Figure 2

Regulation of the expression of genes related to uric acid metabolism. The mRNA expression of uric acid metabolism pathway genes was evaluated by qPCR. (a) Uric acid synthesis genes: ADA and XOD. (b) Uric acid reabsorption genes: GLUT9, URAT1, and UMOD. (c) Uric acid excretion genes: ABCG2 and MRP4. The data are expressed as the mean $\pm S D, n=8$. In $a, b$ and $c$, significant differences $(P<0.05)$ were determined by ANOVA.

\section{Figure 3}

Regulation of inflammatory cytokines. (a) IL-1 $\beta$ and IL- 6 in the liver. (b) TGF- $\beta$ in the liver. (c) IL-1 $\beta$ and IL6 in the kidneys. (d) TGF- $\beta$ in the kidneys. (e) NLRP3 and Caspase- 1 in the kidneys. (f) MyD88 and TRAF6 in the kidneys.

\section{Figure 4}

Influence on the modulation of the gut microbiota structure in hyperuricaemic mice. (a) Classification at the phylum level. (b) Classification at the genus level. (c) Classification at the species level.

\section{Figure 5}

Functional analysis of the intestinal microflora in hyperuric acid mice. (a) KEGG pathway classification. According to KEGG labelling, 405457 unigenes were counted, and the Mann-Whitney U test was performed. Data with FDR values $<0.05$ were selected for plotting. The left vertical axis is the secondary classification information for the KEGG pathway, the right vertical axis is the primary classification information for the KEGG pathway, and the horizontal axis represents the percentage of unigenes annotated. (b) Analysis of KEGG pathways corresponding to the unigenes with differential abundance. Pathway annotation information for the top 30 most abundant KEGG terms in the PathwayEntry database was selected to analyse the differences between the metabolic pathways of hyperuricaemia 
after treatment with S. reilianum polysaccharides and P. dactylifera monosaccharides. (c) Analysis of different unigenes corresponding to strains and pathways.

\section{Figure 6}

PCA of serum metabolite ions in hyperuricaemic mice. Each point in the figure represents a sample, and the similarities and differences among all samples are reflected in the separation and aggregation trends of the points in the figure. Clustering of points indicates that the observed variables have a high degree of similarity, and dispersion of points indicates that the observed variables have obvious differences. (a) PCA in POS mode. (b) PCA in NEG mode.

\section{Figure 7}

Analysis of differential metabolites among groups. Untargeted metabolomics of the samples was performed. After univariate analysis, $T$ tests were used for statistical analysis, and the q values were obtained. For multivariate analysis, PCA and PLS-DA were performed. A ratio $>=2$ or $\langle=2$, a VIP $>=1$ and a q value $<=0.05$ were the screening conditions to obtain significantly different metabolites.

\section{Figure 8}

Analysis of metabolic pathways associated with the serum metabolites of hyperuricaemic mice. (a) Total serum metabolites in the POS model. (b) Total serum metabolites in the NEG model. The first-level KEGG pathway terms are displayed and distinguished by different colours. The second-level KEGG pathway terms are on the abscissa, and the numbers of metabolites are on the ordinate. The identified metabolites were classified into the top 20 KEGG pathways. (c) POS model. (d) NEG model. The x-axis represents the top 20 KEGG pathways, and the $y$-axis represents the number of identified metabolites involved in these pathways.

\section{Figure 9}

Different pathways related to serum metabolites in each group compared with the HF group. (a) Pathway diagram of differential metabolite enrichment in the HF and CT groups. (b) Pathway diagram of differential metabolite enrichment in the HF+AL and HF groups. (c) Pathway diagram of differential metabolite enrichment in the HF+UL and HF groups. (d) Pathway diagram of differential metabolite enrichment in the HF+UH and HF groups. (e) Pathway diagram of differential metabolite enrichment in the HF+DL and HF groups. (f) Pathway diagram of differential metabolite enrichment in the HF+DH and HF groups. 


\section{Supplementary Files}

This is a list of supplementary files associated with this preprint. Click to download.

- Supplementarymaterials1203.doc 